\title{
Perspective Piece \\ Stool-Based Pathogen Detection Offers Advantages as an Outcome Measure for Water, Sanitation, and Hygiene Trials
}

\author{
Joe Brown $^{1 *}$ and Oliver Cumming ${ }^{2}$ \\ ${ }^{1}$ School of Civil and Environmental Engineering, Georgia Institute of Technology, Atlanta, Georgia; ${ }^{2}$ Department of Disease Control, Faculty of \\ Infectious and Tropical Diseases, London School of Hygiene and Tropical Medicine, London, United Kingdom
}

\begin{abstract}
Most health impact trials of water, sanitation, and hygiene use caregiver-reported diarrhea in children as the primary outcome; this measure is known to be subject to considerable bias, especially when used in unblinded trials. Detection of enteric pathogens in stool or fecal waste via multiplex molecular methods may offer advantages over-and is complementary to-caregiver-reported diarrhea because these measures are objective, on the causal pathway from exposures of interest to disease outcomes, and increasingly feasible in high-burden countries.
\end{abstract}

Water, sanitation, and hygiene (WASH) health impact trials have typically used caregiver-reported childhood diarrhea as a primary outcome. This is justified in public health terms as childhood diarrhea is assumed to account for most of the WASH-attributable disease burden. ${ }^{1}$ As a primary outcome for health impact trials, however, diarrhea is problematic as it generally relies on self-reported information, with significant potential for bias, especially when used in unblinded trials. ${ }^{2}$ Beyond these long-standing concerns, important new studies have demonstrated that the etiology of childhood diarrhea in high-burden settings is diverse and varies significantly by setting, population, and $\mathrm{age}^{3}$; moreover, prevalence and detection of a range of enteric pathogen targets is very high in at-risk populations, even in apparently asymptomatic individuals. ${ }^{4}$ Here, we consider stool-based enteric pathogen detection as an alternative, novel outcome for WASH trials, offering several advantages over caregiver-reported diarrhea symptomology.

First, detection of enteric pathogens in stool is an objective outcome measurable in either quantal or quantitative multiplex assays and may include viruses, bacteria, protozoa, and helminths of interest. Such methods are supported by standard, validated procedures used in diagnostic assays, based on wellcharacterized gene targets, offering low detection limits and potential for quantification. ${ }^{5,6} \mathrm{~A}$ range of commercially available kits and custom assays ${ }^{6-8}$ have enabled simultaneous detection of multiple enteric pathogens via molecular methods, many supported by systematic studies reporting sensitivity and specificity, compared with alternative methods in different populations. These are powerful tools widely available for use globally; the required molecular facilities are available in most countries, including those with a high burden of diarrheal disease.

Second, stool-based enteric pathogen detection can identify etiologies of potential importance in populations of interest; such information may assist with developing more effective interventions to reduce exposure. Even in sites where information on the diarrheal disease burden is available, knowledge of potential underlying etiologies of enteric infection and disease remains critically important for the design of interventions to interrupt transmission. Dominant transmission pathways vary by setting and also by pathogen;

*Address correspondence to Joe Brown, School of Civil and Environmental Engineering, Georgia Institute of Technology, 311 Ferst Dr., Atlanta, GA 30332. E-mail: joe.brown@ce.gatech.edu effective interventions to control rotavirus infection in the United States will differ from those for cryptosporidiosis in Kenya.

Third, for an individual, detection of an enteric pathogen in stool is an unambiguous indication of past exposure to that pathogen. Regardless of symptomology and without indicating colonization or potential for current or future effects on gut health, the presence of a pathogen in stool is possible only if the individual has been exposed at some point before the specimen was collected. As WASH interventions are intended to reduce exposure to enteric pathogens, the use of straightforward exposure measures as proximal trial outcomes is justifiable. Enteric pathogen presence in the gut is a necessary precondition of enteric infection, itself on the causal pathway from primary WASH-related or fecal-oral exposure to WASH-related disease outcomes, notably diarrheal diseases, ${ }^{3}$ and various hypothesized sequelae, including environmental enteric dysfunction, ${ }^{9}$ adverse growth ${ }^{10}$ and cognitive impairment, ${ }^{11}$ impacts on the immune system, ${ }^{12}$ and poor oral vaccine performance. ${ }^{13}$

Despite some clear advantages, there are important limitations to stool-based enteric pathogen detection as an outcome measure in trials. The science in this area is evolving rapidly, and our understanding of the many underlying biological mechanisms is incomplete, particularly with respect to the health significance to an individual_-if any_-of shedding pathogens in stool when no symptoms are present. ${ }^{8}$ Pathogen detection in stool may not necessarily indicate an active infection and may not have clear implications for gut health. Although subclinical carriage has been found to be generally high in at-risk populations, ${ }^{3,4}$ enteric pathogens may act more as commensal members of the gut microbiota under some conditions. Enteric pathogen detection may also be inconsistent or ephemeral in stool based on standard diagnostic assays, and persistence of infections may vary according to a range of factors. Enteric pathogen shedding may not be very sensitive to changes in exposure over the relatively short time scales of impact trials; however, more work is needed on asymptomatic shedding of important enteric pathogens in longitudinal cohort studies to provide more evidence on this point. Most methods now under consideration for multiplex pathogen detection in stool are library-dependent, and therefore, detection will be limited to specific targets sought, potentially missing clinically relevant pathogens. Commercially available platforms for identification of enteric pathogens in stool may also lack 
essential transparency in methods, including full reporting of primers and probes that would allow for direct comparison across methods. Metagenomics may offer greater scope for detection of multiple targets, but will generally have a substantially higher limit of detection, lowering sensitivity for individual pathogens of interest, and may be less available in settings where risk is highest. Finally, bulk stool or rectal swab samples may be logistically difficult and, therefore, costly to obtain in large trials, limiting the use of such measures in practice. Using aggregate fecal waste streams, such as fecal sludges or wastewaters, ${ }^{14,15}$ may offer logistical advantages over collection of fecal specimens from individuals; however, this strategy is accompanied by other complications such as pathogen proliferation (for bacteria) and die-off, potentially masking important differences in shedding among individuals, and nonhuman sources. Wastewater monitoring is a common surveillance method supporting, for example, poliovirus eradication programs, but may also be more widely applied among other enteric targets. Whether pathogen detection in environmental matrices can provide a useful proxy outcome measure in trials remains to be seen; more research is required to understand whether and to what extent such signals may be sensitive to changes in disease prevalence or incidence at various scales.

Stool-based enteric pathogen detection offers several advantages over the conventional WASH trial outcome of caregiver-reported diarrhea. Ultimately, without more objective outcomes relating to specific diarrheal etiologies, rather than generalized and subjective symptoms, it will remain challenging to mobilize the most appropriate interventions, whether these be environmental, such as WASH, or medical, such as vaccination.

Received August 28, 2019. Accepted for publication September 24, 2019.

Published online November 4, 2019.

Authors' addresses: Joe Brown, School of Civil and Environmental Engineering, Georgia Institute of Technology, Atlanta, GA, E-mail: joe.brown@ce.gatech.edu. Oliver Cumming, Department of Disease Control, Faculty of Infectious and Tropical Diseases, London School of Hygiene and Tropical Medicine, London, United Kingdom, E-mail: oliver.cumming@Ishtm.ac.uk.

This is an open-access article distributed under the terms of the Creative Commons Attribution (CC-BY) License, which permits unrestricted use, distribution, and reproduction in any medium, provided the original author and source are credited.

\section{REFERENCES}

1. Stanaway JD et al., 2018, Global, regional, and national comparative risk assessment of 84 behavioural, environmental and occupational, and metabolic risks or clusters of risks for 195 countries and territories, 1990-2017: a systematic analysis for the Global Burden of Disease Study 2017. Lancet 392: 1923-1994.

2. Wood L, Egger M, Gluud LL, Schulz KF, Jüni $P$, Altman DG, Gluud C, Martin RM, Wood AJG, Sterne JAC, 2008. Empirical evidence of bias in treatment effect estimates in controlled trials with different interventions and outcomes: metaepidemiological study. BMJ 336: 601-605.

3. Kotloff KL et al., 2013. Burden and aetiology of diarrhoeal disease in infants and young children in developing countries (the Global Enteric Multicenter Study, GEMS): a prospective, casecontrol study. Lancet 382: 209-222.

4. Knee J, Sumner T, Adriano Z, Berendes D, de Bruijn E, Schmidt WP, Nalá R, Cumming O, Brown J, 2018. Risk factors for childhood enteric infection in urban Maputo, Mozambique: a cross-sectional study. PLoS Negl Trop Dis 12: e0006956.

5. Walson JL, Pavlinac PB, 2018. Targeting enteric pathogens to improve childhood survival and growth. Lancet Glob Health 6: e1258-e1259.

6. Liu J et al., 2016 Use of quantitative molecular diagnostic methods to identify causes of diarrhoea in children: a reanalysis of the GEMS case-control study. Lancet 388: 1291-1301.

7. Chhabra $P$ et al., 2017 Comparison of three multiplex gastrointestinal platforms for the detection of gastroenteritis viruses. J Clin Virol 95: 66-71.

8. Freeman K, Mistry H, Tsertsvadze A, Royle P, McCarthy N, TaylorPhillips S, Manuel R, Mason J, 2017. Multiplex tests to identify gastrointestinal bacteria, viruses and parasites in people with suspected infectious gastroenteritis: a systematic review and economic analysis. Health Technol Assess 21: 1-188.

9. Humphrey $\mathrm{JH}, 2009$. Child undernutrition, tropical enteropathy, toilets, and handwashing. Lancet 374: 1032-1035.

10. Rogawski ET et al., 2018 Use of quantitative molecular diagnostic methods to investigate the effect of enteropathogen infections on linear growth in children in low-resource settings: longitudinal analysis of results from the MAL-ED cohort study. Lancet Glob Health 6: e1319-e1328.

11. Stewart CP et al., 2018. Effects of water quality, sanitation, handwashing, and nutritional interventions on child development in rural Kenya (WASH Benefits Kenya): a clusterrandomised controlled trial. Lancet Child Adolesc Health 2: 269-280.

12. Oria RB, Murray-Kolb LE, Scharf RJ, Pendergast LL, Lang DR, Kolling GL, Guerrant RL, 2016. Early-life enteric infections: relation between chronic systemic inflammation and poor cognition in children. Nutr Rev 74: 374-386.

13. Church JA, Parker EP, Kirkpatrick BD, Grassly NC, Prendergast AJ, 2019. Interventions to improve oral vaccine performance: a systematic review and meta-analysis. Lancet Infect Dis 19: 203-214.

14. O’Brien E, Nakyazze J, Wu H, Kiwanuka N, Cunningham W, Kaneene JB, Xagoraraki I, 2017. Viral diversity and abundance in polluted waters in Kampala, Uganda. Water Res 127: 41-49.

15. Falman JC, Fagnant-Sperati CS, Kossik AL, Boyle DS, Meschke JS, 2019. Evaluation of secondary concentration methods for poliovirus detection in wastewater. Food Environ Virol 11: 20-31. 